\title{
Reflexiones sobre Responsabilidad Social en la Empresa Peruana
}

\author{
Reflections on Social Responsibility in the Peruvian Company
}

\section{RESUMEN}

La conceptualización de Responsabilidad Social Empresarial (RSE), o Responsabilidad Social Corporativa (RSC) está referida a las empresas socialmente responsables. El Libro Verde (2001) de la Unión Europea (UE), define a la responsabilidad corporativa como "la integración voluntaria por parte de las empresas, de las preocupaciones sociales y medioambientales en sus operaciones comerciales y sus relaciones con sus interlocutores". Como señala Puterman (2011), los beneficios que se logran cuando una empresa cumple con la responsabilidad social empresarial, son: Transparencia en la gestión, disminución de riesgos, prestigio institucional, retener al mejor talento humano o capital humano, prevenir crisis financieras, crisis sociales, crisis ambientales, reducción de costos fijos, elevar la productividad individual y por ende institucional, mejoramiento continuo, motivación humana, mejora de relaciones con la comunidad, incremento de la rentabilidad, entre otros beneficios.

Palabras claves: Responsabilidad social; medio ambiente; productividad; competitividad.

\begin{abstract}
The conceptualization of Corporate Social Responsibility (CSR) or corporate social responsibility (CSR) refers to socially responsible companies. The Green Book (2001) of the European Union (EU), defines corporate responsibility as "the voluntary integration by companies, social and environmental concerns in their business operations and their relationships with their interlocutors"

As Puterman (2011) points out, the benefits of CSR corporate image and business reputation, reducing operating costs, strengthening the capacity of organizations to recruit and retain top employees, strengthening the relationship is the community, and its environment, the strengthening of consumer loyalty, the improvement of quality and productivity, and the increase in profitability, among many others.
\end{abstract}

Keywords: Social responsibility; environment; productivity; competitiveness.

(C) Los autores. Este artículo es publicado por Gestión en el Tercer Milenio de la Facultad de Ciencias Administrativas de la Universidad Nacional Mayor de San Marcos. Este es un artículo de acceso abierto, distribuido bajo los términos de la licencia Creative Commons Atribucion - No Comercia_Compartir Igual 4.0 Internacional. (http://creativecommons.org/licenses/by-nc-sa/4.0/) que permite el uso no comercial, distribución y reproducción en cualquier medio, siempre que la obra original sea debidamente citada. 


\section{INTRODUCCIÓN}

Las empresas deben considerar a la RSE como una cultura empresarial fomentando acciones estratégicas de responsabilidad social en respuesta a las presiones de la sociedad con respecto a la preservación y conservación del medio ambiente y a la situación económica y social. La RSE es una filosofía de vida que busca una interacción entre los trabajadores, accionistas, clientes, proveedores, autoridades políticas y organizaciones no gubernamentales (ONG), para planificar e invertir en su futuro e incluso llegar a incrementar su rentabilidad. Una empresa que asume una responsabilidad social realiza prácticas correctas en el aprendizaje continuo, organizando mejor el trabajo, ofreciendo mayores oportunidades, ofertando más empleo y logrando un desarrollo sostenible. Las empresas que asumen la RSE elevan sus niveles de desarrollo social, previenen y conservan el medio ambiente, respetan los derechos humanos de acuerdo a la declaración de las naciones unidas, y aplican un modo de gestión y gobierno que armoniza intereses de todos los públicos dentro de un enfoque global fomentando la calidad y la posibilidad de competir a nivel global.

Se puede decir que la RSE se conceptúa también como la actitud y el conocimiento de una empresa que se encamina a sostener el equilibrio entre el desarrollo social, desarrollo sustentable, y crecimiento económico de ella misma. La responsabilidad social se puede considerar como una inversión más no un gasto, es igual a que se apostara por el mejoramiento de la calidad. Su objetivo principal es lograr objetivos sociales, económicos y medioambientales y como beneficios se mejora la imagen institucional corporativa, simplificación de costos operativos, mantener a los mejores talentos, fortalecer los lazos con la sociedad, satisfacer a los clientes, mejoramiento continuo de la calidad y elevar los índices de productividad, así como mejorar los beneficios económicos. El libro verde que la comunidad económica europea manifiesta:

No se debe considerar sustitutiva de la reglamentación o legislación sobre los derechos sociales o normas medioambientales, ni permite soslayar la elaboración de nuevas normas apropiadas. En los países que carecen de tales reglamentaciones, los esfuerzos deben centrarse en la instauración del marco legislativo o reglamentario adecuado, a fin de definir un entorno uniforme a partir del cual desarrollar prácticas socialmente responsables.

Podemos decir que cualquier empresa debe asumir su responsable social para que su gestión refleje una imagen positiva tanto interna como externa, transparentando su nivel ético.

\section{OBJETIVO}

Estas reflexiones tienen como objetivo principal la conceptualización, interpretación y entendimiento de la RSE por parte de los empresarios para que sus organizaciones pongan en práctica esta filosofía de vida y comportamiento institucional, para llegar a un mayor compromiso social en el manejo del medio ambiente, otorgar mayores satisfacciones a sus trabajadores, respetar los derechos humanos, mantener y estrechar más los vínculos con los stakeholders, aumentar la productividad, mejorar la calidad y mayores índices de rentabilidad. La RSE permite que las empresas sean más competitivas, sepan sobrevivir en la adversidad y logren con eficiencia la visión y misión en sus planes estratégicos.

\section{ARGUMENTACIÓN}

La RSE en su aplicación se fundamenta en una economía basada en el conocimiento que le permita ser más competitiva y dinámica, que tenga la capacidad de responder a un desarrollo económico sostenible, creando más empleabilidad, y mantener tecnologías limpias para evitar la contaminación del medio ambiente. La gestión de la RSE en las organizaciones y sus relaciones con los públicos son cambiantes en función a sus diferencias culturales y sectoriales. Estas empresas se obligan a declarar algunos principios, valores, código de ética, objetivos, y sus responsabilidades se coordinan con sus interlocutores, estos valores y objetivos son incorporados a sus planes estratégicos y a la toma de decisiones rutinarias y se obligan a efectuar una gestión social y ecológica y posteriormente se evalúan los resultados para medir las acciones sociales y medioambientales y establecer el desarrollo de programas de capacitación y formación continua.

El Libro Verde de la comunidad económica europea señala como aspecto fundamental que 
la Responsabilidad Social Empresarial es un deber y un derecho social.

Bestratén y Pujol (2004), manifiestan que una empresa asume la responsabilidad social cuando:

- Los productos y servicios satisfacen plenamente las necesidades de los usuarios, logrando su bienestar tanto en las condiciones y calidad de vida.

- Demuestran una conducta ética en su gestión.

- Tiene programas que evita cualquier riesgo que atente contra la salud y seguridad de sus empleados.

- Desarrolla programas para cumplir con las normas de preservación y conservación del medio ambiente, y mantiene excelentes relaciones con la comunidad.

Existen otras investigaciones sobre responsabilidad social empresarial que han llegado a la conclusión de que una empresa tiene responsabilidad social si cumple con las siguientes condiciones:

- Cuando tiene ética institucional

- No contamina el medio ambiente

- Mantiene un compromiso con la comunidad

- Mantiene política responsable de marketing

La Asociación Nacional de Industriales de Colombia, en su revista ANDI, manifiesta que una empresa es responsable socialmente cuando contribuye al desarrollo social y al bienestar de sus empleados y la comunidad en general.

La responsabilidad social como proceso de gestión, se tiene que concebir como una serie de fases integrado que conlleva a fines integrados en la planeación estratégica. Se ha señalado anteriormente, Bestratén y Pujol (2004), que señalan tres categorías que deben garantizar el cumplimiento de acciones responsables: Primarias, Secundarias, y Terciarias.
1. Las responsabilidades primarias, son consideradas como la razón de ser de la empresa, no son acciones estratégicas de responsabilidad social. Estas responsabilidades incluyen: Brindar productos y servicios de calidad con precios justos, el respeto a los derechos laborales de los trabajadores mejorando sus condiciones de trabajo, capacitación permanente para el desarrollo personal y profesional, lograr una empresa altamente competitiva, rentable y sostenible, prevenir los impactos negativos sobre el medio ambiente, cumplir estrictamente las leyes y dispositivos legales que sean aplicables a la gestión empresarial, y ser justa y equitativa en la distribución de la riqueza.

2. Las responsabilidades secundarias, son acciones de gestión que sobre pasan las obligaciones de la empresa originando un gran esfuerzo. Bestratén y Pujol (2004) clasifican a la responsabilidad social, aquellas que tiene que respetar los derechos de los trabajadores y aquellas que se relacionan con la sociedad. Con respecto a los trabajadores: Ofrecer un trabajo digno con buenas condiciones de calidad y seguridad; fomentar la creatividad, innovación y autonomía en su desempeño; garantizarles estabilidad laboral; lograr un alto desarrollo personal y profesional en sus trabajadores; brindar un seguro de salud familiar; reconocer los esfuerzos y resultados alcanzados; desarrollar programas de motivación e incentivos para los más talentosos.

Con respecto a la sociedad: Generar empleo en la comunidad; mantener el medio ambiente descontaminado; efectuar campañas de publicidad comercial de sus productos, valores, en su entorno con un enfoque más humano; integrar a su personal con capacidades y habilidades especiales; coordinar con los proveedores para mejorar las relaciones y los servicios, brindar a la comunidad asesoría en temas especializados que tenga la empresa; pre- 
star colaboración en el desarrollo de los nuevos jóvenes profesionales.

\section{Las responsabilidades terciarias,} son acciones que no se consideran en las actividades propias de la organización, están ligadas con el aspecto social y tienen un impacto en el entorno. Entre estas acciones se pueden señalar: Apoyar a la población en todo tipo de actividades socioeconómicas; proporcionar ayuda a la población para mejora de viviendas; ayudar a la comunidad a la realización de eventos culturales y recreativos; prestar colaboración a la comunidad para mejorar escuelas, universidades o cualquier otra institución de carácter educativo; prestar apoyo a la estructura social de la comunidad.

Los problemas medioambientales se relacionan con las empresas y a su consumo de los recursos naturales que disponen los países del mundo entero. Frente a esta problemática las empresas deben fomentar la reducción del impacto ambiental en su cadena de producción, en el marco de políticas integradas de productos (PIP). Por lo tanto, la RSE implica cambios en la gestión estratégica, que según Solano (2005), esta gestión debe tener las condiciones y características siguientes:

- Objetivos definidos y determinísticos claros respecto a las soluciones a que se quiere llegar. Estos objetivos deben consensualizarse y compartirse.

- Promueve el cambio.

- Medios de comunicación para difundir e implementar un programa de cambios.

- Desarrollo de estrategias que promuevan una planificación hacia el cambio continuo.

- Desarrollo de estrategias sustentables, en el tiempo.

\section{Según Pizzolante Negrón (2009):}

Para ser líder en sustentabilidad se tiene que minimizar nuestras las huellas del pasado, y maximizar los aportes y contribuciones a la sociedad, fortaleciendo los niveles de competitividad con una buena planificación estratégica debidamente consensuada logrando que el mundo actual se convierta en un lugar donde aprendamos a vivir juntos a través del tiempo.

En cuanto a la gestión del impacto ambiental y el uso de los recursos naturales se consideran como inversiones tanto para las organizaciones como para la conservación del medio ambiente natural. Este enfoque se considera como un principio reconocido por la UE para ayudar a las empresas a detectar oportunidades de negocios y a realizar inversiones que redunden en beneficio para las partes, es decir, tanto para la empresa como para sociedad. Los programas para la gestión del impacto ambiental, conservación del medio ambiente y la sustentabilidad o desarrollo sustentable en su contenido debe considerar: armonización a nivel nacional de todas las empresas, medidas efectivas y respetuosas con la contaminación del medio ambiente, y reconocimiento a las empresas que obtengan buenos resultados. El problema ambiental, incluye algunos componentes abióticos como el aire, suelo, agua, luz, seres vivos de distintas especies, entre animales y vegetales y el hombre con su cultura. La contaminación del medio ambiente es originada por las alteraciones causadas por las actividades humanas o condiciones naturales de cambios en el medio que el hombre tiene que solucionar para obtener una mejor calidad de vida.

La vida del ser humano depende de la naturaleza, y constantemente la modifica más que otras especies, por su inteligencia y capacidad cultural.

Este escenario donde vive el ser humano le permite hacer uso de sus recursos naturales para la sobrevivencia y mejorar sus condiciones de vida. Los recursos naturales que se emplean como insumos en las empresas para la producción de bienes, actualmente sufren una sobreexplotación indiscriminada que pone en riesgo a todos los seres vivos del planeta. El mayor impacto de la sobreexplotación de los recursos naturales es la desaparición de la biodiversidad, es decir, la extinción de algunos recursos que tienen como consecuencia el deterioro ambiental y pérdida de una fuente importante de ingresos en la vida socioeconómica. Los gobiernos deben establecer políticas para 
proteger el uso irracional de los recursos naturales, específicamente sobre los no renovables. Los efectos y daños de la sobreexplotación de los recursos se muestran en la disminución de superficies utilizables entre cinco y siete millones de hectáreas al año, la contaminación de ríos, mares y lagos que acaban con la vida de muchas especies, agotamientos de minerales y combustibles fósiles, la tala de los bosques que da lugar a la deforestación que afecta un medio de vida para mucha gente, la sobreexplotación de las especies marinas que puede traer consecuencias muy graves en la vida de los seres humanos ya que la plataforma marina es considerada como la despensa de la humanidad, la caza y el comercio de especies protegidas que ponen en peligro algunas especies en vías de extinción, la sobreexplotación del petróleo (hidrocarburos), en su estado sólido, líquido y gaseoso. Frente a todo este siniestro panorama se deben establecer algunas medidas preventivas para evitar o minimizar los efectos generados por la inhumana sobreexplotación, destrucción de los recursos naturales y extinción de algunas especies. y también para recuperar parcial o totalmente algunos recursos.

La RSE en el Perú, no ha logrado a crear una conciencia empresarial, ni leyes que respondan a las exigencias en cuanto a responsabilidad empresarial como viene sucediendo en otros países de América Latina. Se necesita un marco legal para establecer una obligatoriedad de las empresas a asumir su responsabilidad social como un instrumento para proteger los derechos de los públicos (stakeholders) con los cuales las empresas mantienen relaciones o transacciones comerciales, en este sentido se logra satisfacer a los usuarios y consumidores con un alto espíritu de equidad social, conservando el medio ambiente como una herramienta para el desarrollo empresarial.

En el Perú, en estos últimos años muchas empresas han despertado el interés en el sentido de que la RSE debe implementarse en las organizaciones, pero ha requerido difusión y sensibilización estratégica para comprender esta nueva corriente de pensamiento empresarial. Actualmente, existe en el Perú un programa "Perú Responsable" a cargo del Ministerio de Trabajo y Promoción del empleo que promueve ideas y conceptos para crear una cultura de re- sponsabilidad social empresarial en los diferentes sectores públicos. La RSE en las empresas extractivas ha desarrollado campañas de difusión en las localidades indígenas para obligar a estas comunidades para que con sus propios esfuerzos alcancen el desarrollo sostenible con la ayuda económica que la empresa les pueda alcanzar.

El Estado peruano tiene alianzas estratégicas con algunas empresas del sector privado para lograr un mayor impacto en lo relacionado con la RSE. Cabe resaltar que la Superintendencia de Mercado de Valores está exigiendo a las empresas reportar en sus memorias anuales que acciones de sostenibilidad han realizado. Se han efectuado encuestas en el Perú a cien (100) CEOS, de los cuales el 71 por ciento indican que la sostenibilidad es un nuevo paradigma para que las empresas se desarrollen e implanten programas de innovación de bienes y servicios. En la encuesta realizada en el año 2013, el 26 por ciento de las organizaciones de una población de 140 manifestaron que desarrollan programas de RSE. Durante el año 2014, el 58 por ciento señaló que sus negocios ya estaban implementándose. Al final el estudio también indicaba que la RSE es considerada como un tema importante y trascendente. En el aspecto evaluativo, el 62 por ciento de los CEOS consideran como regular las actividades de responsabilidad social empresarial, sin embargo, el 31 por ciento consideran que los programas de RSE son buenos. El paradigma de la RSE en el Perú es un desafío permanente que cada vez cobra más relevancia y trascendencia, y que ponerla en práctica comprende una serie de lineamientos estratégicos que deben ser incorporados a los planes estratégicos de las empresas, para ello se hace necesario una coordinación entre el Estado, las instituciones académicas universitarias, la prensa hablada y escrita y la sociedad civil quien debe asumir una actitud positiva, proactiva y propositiva.

Hasta la década de los 80 , la RSE se convierte en una preocupación que involucra a numerosos actores entre los que están comprendidos no solamente a las empresas y diferentes grupos de consumidores, sino también a la comunidad científica, gobernantes, ONG, políticos, intelectuales, sociedad civil y organismos internacionales. En este nuevo escenario apare- 
cen algunas teorías que confirman el interés y preocupación internacional de la RSE y en los años 90. Garriga y Melé (2004) clasifican en cuatro grandes grupos:

a. Las Instrumentales, considera que las empresas se relacionan exclusivamente con el mercado y que su funcionalidad es obtener un beneficio económico sirviendo a la sociedad, es decir, su función fundamental es crear valor y riqueza a través de los bienes y servicios que demandan los mercados.

b. Las políticas, esta teoría concibe a las empresas como organizaciones que tienen poder de decisión y que ésta se extrae de sus directorios que es el gobierno de la corporación.

c. Las Integradoras, estas teorías sostienen que la empresa capta y responde directamente a las demandas de la sociedad con el objeto de legitimarse y prestigiarse socialmente.

Este grupo de teorías comprende

1. La gestión de asuntos sociales, viene a ser la respuesta a las demandas sociales y políticas que pueden afectarla seriamente en su funcionamiento.

2. El principio de responsabilidad pública, se enfoca en que el comportamiento de una empresa se deriva de una política pública relevante, que atiende los reclamos de la opinión pública en asuntos emergentes, legales, formales y prácticos.

3. La gestión de los grupos implicados (stakeholders), se orienta a las personas que pueden ser afectadas por las políticas y prácticas corporativas, su ventaja es que se relaciona con su entorno y comprende mejor a los agentes de las situaciones que afronta la empresa.

4. La acción social corporativa, implica algunas teorías que buscan la legitimidad social a través de procesos que responden adecuadamente a las demandas sociales. Las éticas, se fundamentan en la relación que tienen las empresas con la ética que demanda la sociedad, es decir, lo ético está por encima de cualquier otro interés.

\section{CONCLUSIONES}

La RSE debe ser considerada como parte de la cultura empresarial logrando posicionarse en la mente de los empresarios, accionistas, profesionales, técnicos, expertos y todos los demás integrantes de la organización, porque la RSE es tarea de todos. La sociedad demanda de las organizaciones empresariales el uso de tecnologías limpias para evitar la contaminación del medio ambiente y el uso racional de los recursos para un desarrollo social sustentable y garantizar de esta manera un futuro mejor a las generaciones venideras. La RSE permite lograr mayores niveles de desarrollo social, previene y conserva el medio ambiente, respeta los derechos humanos, aplica una gestión y gobierno que armoniza intereses de los stakeholders y así elevar los de competitividad en el mercado global. Las empresas que cumplen con la filosofía de la RSE no realizan gastos, sino, inversiones con el mismo criterio cuando se invierte para el mejoramiento de la calidad y mejorar la productividad individual.

Se considera a la RSE como un conjunto de prácticas internas y externas axiológicamente transparentes y éticas que le van a permitir una mejora en los beneficios económicos que conllevan crecimiento y desarrollo empresarial.

\section{AGRADECIMIENTOS Y RECONOCIMIENTOS}

A Clhoe, Natalie, Briana, Haidy, Vanessa y Enny por ser la energía inspiradora y a la FCA de la UNMSN por brindarme la oportunidad de expresar mis conocimientos y experiencias.

\section{REFERENCIAS BIBLIOGRÁFICAS}

Bestratén y Pujol (2014), Márkov decisión processes. Revista Discrete

Solano Cornejo, David (2009). Responsabilidad Social: Estrategias sostenibles para el desarrollo y la competitividad. Editorial ESAN. 
Garriga y Melé (2004). Revista comunica RSE. Comunicación de Responsabilidad \& Sustentabilidad Empresaria. Artículo de opinión. - Mapa de teorías de RSE (2004).

Almagro, Juan; Garmendia, José Antonio; De la Torre, Isabel (2010). Responsabilidad Social: Una reflexión global sobre la RSE.

Iturregui \& Perla- Comunicación, ambiente y desarrollo SAC. Edit. Libélula (2016)

La Revista, Acción RSE (2001). Revista de Negocios Internacionales (2001). Universidad EAFIT.
Pizzolante Negrón /2009). De la Responsabilidad Social Empresarial a la Empresa Socialmente Responsable. Edit.Ciencias Sociales.

Puterman (2011). Markov Decisión Processes: Discrete (2014).

Saenz Acosta, César (2012). ISO 26000: Guía de Responsabilidad Social. Editorial CENGAGE.

Rojas Forero, Geisler Dayani (2017). Gerencia de la Responsabilidad Social en las organizaciones de hoy. Edit. Apfaomega. 
